\title{
SUBORDINATION BY CONVEX FUNCTIONS
}

\author{
ROSIHAN M. ALI, V. RAVICHANDRAN, AND N. SEENIVASAGAN
}

Received 30 July 2006; Accepted 12 November 2006

For a fixed analytic function $g(z)=z+\sum_{n=2}^{\infty} g_{n} z^{n}$ defined on the open unit disk and $\gamma<1$, let $T_{g}(\gamma)$ denote the class of all analytic functions $f(z)=z+\sum_{n=2}^{\infty} a_{n} z^{n}$ satisfying $\sum_{n=2}^{\infty}\left|a_{n} g_{n}\right| \leq 1-\gamma$. For functions in $T_{g}(\gamma)$, a subordination result is derived involving the convolution with a normalized convex function. Our result includes as special cases several earlier works.

Copyright (c) 2006 Hindawi Publishing Corporation. All rights reserved.

\section{Introduction}

Let $\mathscr{A}$ be the class of all normalized analytic functions of the form

$$
f(z)=z+\sum_{n=2}^{\infty} a_{n} z^{n} \quad(z \in \Delta:=\{z \in \mathbb{C}:|z|<1\}) .
$$

Let $S^{*}(\alpha)$ and $C(\alpha)$ be the usual classes of normalized starlike and convex functions of order $\alpha$, respectively, and let $C:=C(0)$. For $f(z)$ given by $(1.1)$ and $g(z)$ by

$$
g(z)=z+\sum_{n=2}^{\infty} g_{n} z^{n}
$$

the convolution (or Hadamard product) of $f$ and $g$, denoted by $f * g$, is defined by

$$
(f * g)(z):=z+\sum_{n=2}^{\infty} a_{n} g_{n} z^{n}
$$

The function $f(z)$ is subordinate to the function $g(z)$, written as $f(z) \prec g(z)$, if there is an analytic function $w(z)$ defined on $\Delta$ with $w(0)=0$ and $|w(z)|<1$ such that $f(z)=$ $g(w(z))$. 
Let $g(z)$ given by (1.2) be a fixed function, with $g_{n} \geq g_{2}>0(n \geq 2), \gamma<1$, and let

$$
T_{g}(\gamma):=\left\{f(z) \in \mathscr{A}: \sum_{n=2}^{\infty}\left|a_{n} g_{n}\right| \leq 1-\gamma\right\} .
$$

The class $T_{g}(\gamma)$ includes as its special cases various other classes that were considered in several earlier works. In particular, for $\gamma=\alpha$ and $g_{n}=n-\alpha$, we obtain the class $\operatorname{TS}^{*}(\alpha):=$ $T_{g}(\gamma)$ that was introduced by Silverman [6]. Putting $\gamma=\alpha$ and $g_{n}=n(n-\alpha)$, we get $T C(\alpha):=T_{g}(\gamma)$. For these classes, Silverman [6] proved that $T S^{*}(\alpha) \subseteq S^{*}(\alpha)$ and $T C(\alpha)$ $\subseteq C(\alpha)$.

By using convolution, Ruscheweyh [5] defined the operator

$$
D^{\alpha} f(z):=\frac{z}{(1-z)^{\alpha+1}} * f(z) \quad(\alpha>-1) .
$$

Let $R_{\alpha}(\beta)$ denote the class of functions $f(z)$ in $\mathscr{A}$ that satisfies the inequality

$$
\mathfrak{R} \frac{D^{\alpha+1} f(z)}{D^{\alpha} f(z)}>\frac{\alpha+2 \beta}{2(\alpha+1)} \quad(\alpha \geq 0,0 \leq \beta<1, z \in \Delta)
$$

Al-Amiri [1] called functions in this class as prestarlike functions of order $\alpha$ and type $\beta$. Let $H_{\alpha}(\beta)$ denote the class of functions $f(z)$ given by (1.1) whose coefficients satisfy the condition

$$
\sum_{n=2}^{\infty}(2 n+\alpha-2 \beta) C(\alpha, n)\left|a_{n}\right| \leq 2+\alpha-2 \beta \quad(\alpha \geq 0,0 \leq \beta<1)
$$

where

$$
C(\alpha, n):=\prod_{k=2}^{n} \frac{(k+\alpha-1)}{(n-1) !} \quad(n=2,3, \ldots) .
$$

Al-Amiri [1] proved that $H_{\alpha}(\beta) \subseteq R_{\alpha}(\beta)$. By taking $g_{n}=(2 n+\alpha-2 \beta) C(\alpha, n)$ and $\gamma=$ $2 \beta-1-\alpha$, we see that $H_{\alpha}(\beta):=T_{g}(\gamma)$.

For functions in the class $H_{\alpha}(\beta)$, Attiya [2] proved the following.

Theorem 1.1 [2, Theorem 2.1, page 3]. If $f(z) \in H_{\alpha}(\beta)$ and $h(z) \in \mathscr{C}$, then

$$
\begin{gathered}
\frac{(4+\alpha-2 \beta)(1+\alpha)}{2[\alpha+(2+\alpha)(3+\alpha-2 \beta)]}(f * h)(z) \prec h(z), \\
\Re(f(z))>-\frac{\alpha+(2+\alpha)(3+\alpha-2 \beta)}{(4+\alpha-2 \beta)(1+\alpha)} .
\end{gathered}
$$

The constant factor

$$
\frac{(4+\alpha-2 \beta)(1+\alpha)}{2[\alpha+(2+\alpha)(3+\alpha-2 \beta)]}
$$

in the subordination result (1.9) cannot be replaced by a larger number. 
Owa and Srivastava [4] as well as Owa and Nishiwaki [3] studied the subclasses $M^{*}(\alpha)$ and $\mathcal{N}^{*}(\alpha)$ consisting of functions $f \in \mathscr{A}$ satisfying

$$
\begin{aligned}
& \sum_{n=2}^{\infty}[n-\lambda+|n+\lambda-2 \alpha|]\left|a_{n}\right| \leq 2(\alpha-1) \quad(\alpha>1,0 \leq \lambda \leq 1), \\
& \sum_{n=2}^{\infty} n[n-\lambda+|n+\lambda-2 \alpha|]\left|a_{n}\right| \leq 2(\alpha-1) \quad(\alpha>1,0 \leq \lambda \leq 1),
\end{aligned}
$$

respectively. These are special cases of $T_{g}(\gamma)$, with $g_{n}=n-\lambda+|n+\lambda-2 \alpha|, \gamma=3-2 \alpha$, and $g_{n}=n(n-\lambda+|n+\lambda-2 \alpha|), \gamma=3-2 \alpha$, respectively. For the class $\mu^{*}(\alpha)$, Srivastava and Attiya [8] proved the following.

Theorem $1.2\left[8\right.$, Theorem 1, page 3]. Let $f(z) \in \mathcal{M}^{*}(\alpha)$. Then for any function $h(z) \in C$ and $z \in \Delta$,

$$
\begin{gathered}
\frac{2-\lambda+|2+\lambda-2 \alpha|}{2[2 \alpha-\lambda+|2+\lambda-2 \alpha|]}(f * h)(z) \prec h(z), \\
\Re(f(z))>-\frac{2 \alpha-\lambda+|2+\lambda-2 \alpha|}{[(2-\lambda)+|2+\lambda-2 \alpha|]} .
\end{gathered}
$$

The constant factor

$$
\frac{2-\lambda+|2+\lambda-2 \alpha|}{2[2 \alpha-\lambda+|2+\lambda-2 \alpha|]}
$$

in the subordination result (1.13) cannot be replaced by a larger number.

A similar result $\left[8\right.$, Theorem 2, page 5] for $\mathcal{N}^{*}(\alpha)$ was also obtained.

In this article, Theorems 1.1 and 1.2 are unified for the class $T_{g}(\gamma)$. Relevant connections of our results with several earlier investigations are also indicated.

We need the following result on subordinating factor sequence to obtain our main result. Recall that a sequence $\left(b_{n}\right)_{1}^{\infty}$ of complex numbers is said to be a subordinating factor sequence, if for every convex univalent function $f(z)$ given by (1.1), then

$$
\sum_{n=1}^{\infty} a_{n} b_{n} z^{n} \prec f(z) .
$$

Theorem 1.3 [9, Theorem 2, page 690]. A sequence $\left(b_{n}\right)_{1}^{\infty}$ of complex numbers is a subordinating factor sequence if and only if

$$
\mathfrak{R}\left(1+2 \sum_{n=1}^{\infty} b_{n} z^{n}\right)>0
$$


4 Subordination by convex functions

\section{Subordination with convex functions}

We begin with the following subordination result.

Theorem 2.1. If $f(z) \in T_{g}(\gamma)$ and $h(z) \in C$, then

$$
\begin{gathered}
\frac{g_{2}}{2\left(g_{2}+1-\gamma\right)}(f * h)(z) \prec h(z), \\
\mathfrak{R}(f(z))>-\frac{g_{2}+1-\gamma}{g_{2}} \quad(z \in \Delta) .
\end{gathered}
$$

The constant factor

$$
\frac{g_{2}}{2\left(g_{2}+1-\gamma\right)}
$$

in the subordination result (2.1) cannot be replaced by a larger number.

Proof. Let $G(z)=z+\sum_{n=2}^{\infty} g_{2} z^{n}$. Since $T_{g}(\gamma) \subseteq T_{G}(\gamma)$, our result follows if we prove the result for the class $T_{G}(\gamma)$. Let $f(z) \in T_{G}(\gamma)$ and suppose that

$$
h(z)=z+\sum_{n=2}^{\infty} c_{n} z^{n} \in C .
$$

In this case,

$$
\frac{g_{2}}{2\left(g_{2}+1-\gamma\right)}(f * h)(z)=\frac{g_{2}}{2\left(g_{2}+1-\gamma\right)}\left(z+\sum_{n=2}^{\infty} c_{n} a_{n} z^{n}\right) .
$$

Observe that the subordination result (2.1) holds true if

$$
\left(\frac{g_{2}}{2\left(g_{2}+1-\gamma\right)} a_{n}\right)_{1}^{\infty}
$$

is a subordinating factor sequence (with of course, $a_{1}=1$ ). In view of Theorem 1.3 , this is equivalent to the condition that

$$
\mathfrak{R}\left\{1+\sum_{n=1}^{\infty} \frac{g_{2}}{g_{2}+1-\gamma} a_{n} z^{n}\right\}>0 .
$$

Since $g_{n} \geq g_{2}>0$ for $n \geq 2$, we have

$$
\begin{aligned}
\mathfrak{R}\left\{1+\frac{g_{2}}{g_{2}+1-\gamma} \sum_{n=1}^{\infty} a_{n} z^{n}\right\} & =\mathfrak{R}\left\{1+\frac{g_{2}}{g_{2}+1-\gamma} z+\frac{1}{g_{2}+1-\gamma} \sum_{n=2}^{\infty} g_{2} a_{n} z^{n}\right\} \\
& \geq 1-\left\{\frac{g_{2}}{g_{2}+1-\gamma} r+\frac{1}{g_{2}+1-\gamma} \sum_{n=2}^{\infty}\left|g_{2} a_{n}\right| r^{n}\right\} \\
& >1-\left\{\frac{g_{2}}{g_{2}+1-\gamma} r+\frac{1-\gamma}{g_{2}+1-\gamma} r\right\}>0 \quad(|z|=r<1) .
\end{aligned}
$$


Rosihan M. Ali et al. 5

Thus (2.7) holds true in $\Delta$, and proves (2.1). The inequality (2.2) follows by taking $h(z)=$ $z /(1-z)$ in $(2.1)$.

Now consider the function

$$
F(z)=z-\frac{1-\gamma}{g_{2}} z^{2} \quad(\gamma<1) .
$$

Clearly, $F(z) \in T_{g}(\gamma)$. For this function $F(z)$, (2.1) becomes

$$
\frac{g_{2}}{2\left(g_{2}+1-\gamma\right)} F(z) \prec \frac{z}{1-z} \text {. }
$$

It is easily verified that

$$
\min \left\{\mathfrak{R}\left(\frac{g_{2}}{2\left(g_{2}+1-\gamma\right)} F(z)\right)\right\}=-\frac{1}{2} \quad(z \in \Delta) .
$$

Therefore the constant

$$
\frac{g_{2}}{2\left(g_{2}+1-\gamma\right)}
$$

cannot be replaced by any larger one.

Corollary 2.2. If $f(z) \in T S^{*}(\alpha)$ and $h(z) \in C$, then

$$
\frac{2-\alpha}{2(3-2 \alpha)}(f * h)(z) \prec h(z), \quad \Re(f(z))>-\frac{3-2 \alpha}{2-\alpha} \quad(z \in \Delta) .
$$

The constant factor

$$
\frac{2-\alpha}{2(3-2 \alpha)}
$$

in the subordination result (2.13) cannot be replaced by a larger number.

Remark 2.3. The case $\alpha=0$ in Corollary 2.2 was obtained by Singh [7].

Corollary 2.4. If $f(z) \in T C(\alpha)$ and $h(z) \in C$, then

$$
\frac{2-\alpha}{5-3 \alpha}(f * h)(z) \prec h(z), \quad \mathfrak{R}(f(z))>-\frac{5-3 \alpha}{2(2-\alpha)} \quad(z \in \Delta) .
$$

The constant factor

$$
\frac{2-\alpha}{5-3 \alpha}
$$

in the subordination result (2.15) cannot be replaced by a larger one.

Remark 2.5. Theorem 1.1 is obtained by taking $\gamma=2 \beta-1-\alpha$ and

$$
g_{n}=(2 n+\alpha-2 \beta) \prod_{k=2}^{n} \frac{(k+\alpha-1)}{(n-1) !} \quad(n=2,3 \ldots, \alpha>0,0 \leq \beta<1)
$$


in Theorem 2.1. Similarly, putting $\gamma=3-2 \alpha$ and

$$
g_{n}=n-\lambda+|n+\lambda-2 \alpha| \quad(n=2,3 \ldots, \alpha>1,0 \leq \lambda \leq 1)
$$

in Theorem 2.1 yields Theorem 1.2. Finally, by taking $\gamma=3-2 \alpha$ and

$$
g_{n}=n(n-\lambda+|n+\lambda-2 \alpha|) \quad(n=2,3 \ldots, \alpha>1,0 \leq \lambda \leq 1)
$$

in Theorem 2.1, we get [8, Theorem 2, page 5].

\section{Acknowledgment}

The authors gratefully acknowledged support from IRPA Grant 09-02-05-00020 EAR.

\section{References}

[1] H. S. Al-Amiri, Prestarlike functions of order $\alpha$ and type $\beta$ with negative coefficients, Annales Universitatis Mariae Curie-Skłodowska. Sectio A. Mathematica 39 (1985), 1-11 (1988).

[2] A. A. Attiya, On some applications of a subordination theorem, Journal of Mathematical Analysis and Applications 311 (2005), no. 2, 489-494.

[3] S. Owa and J. Nishiwaki, Coefficient estimates for certain classes of analytic functions, Journal of Inequalities in Pure and Applied Mathematics 3 (2002), no. 5, 5, article 72.

[4] S. Owa and H. M. Srivastava, Some generalized convolution properties associated with certain subclasses of analytic functions, Journal of Inequalities in Pure and Applied Mathematics 3 (2002), no. 3,13 , article 42 .

[5] S. Ruscheweyh, New criteria for univalent functions, Proceedings of the American Mathematical Society 49 (1975), no. 1, 109-115.

[6] H. Silverman, Univalent functions with negative coefficients, Proceedings of the American Mathematical Society 51 (1975), no. 1, 109-116.

[7] S. Singh, A subordination theorem for spirallike functions, International Journal of Mathematics and Mathematical Sciences 24 (2000), no. 7, 433-435.

[8] H. M. Srivastava and A. A. Attiya, Some subordination results associated with certain subclasses of analytic functions, Journal of Inequalities in Pure and Applied Mathematics 5 (2004), no. 4, 6, article 82.

[9] H. S. Wilf, Subordinating factor sequences for convex maps of the unit circle, Proceedings of the American Mathematical Society 12 (1961), no. 5, 689-693.

Rosihan M. Ali: School of Mathematical Sciences, Universiti Sains Malaysia (USM),

Penang 11800, Malaysia

E-mail address: rosihan@cs.usm.my

V. Ravichandran: School of Mathematical Sciences, Universiti Sains Malaysia (USM), Penang 11800, Malaysia

E-mail address: vravi@cs.usm.my

N. Seenivasagan: School of Mathematical Sciences, Universiti Sains Malaysia (USM), Penang 11800, Malaysia

E-mail address: vasagan2000@yahoo.co.in 


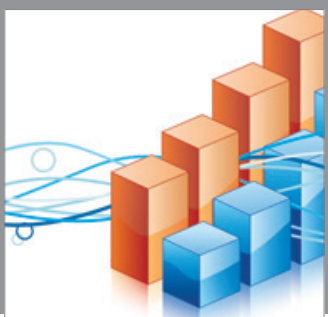

Advances in

Operations Research

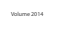

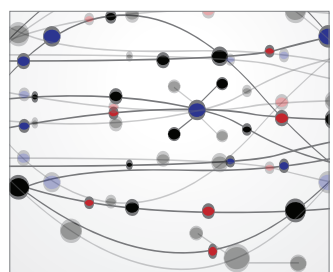

\section{The Scientific} World Journal
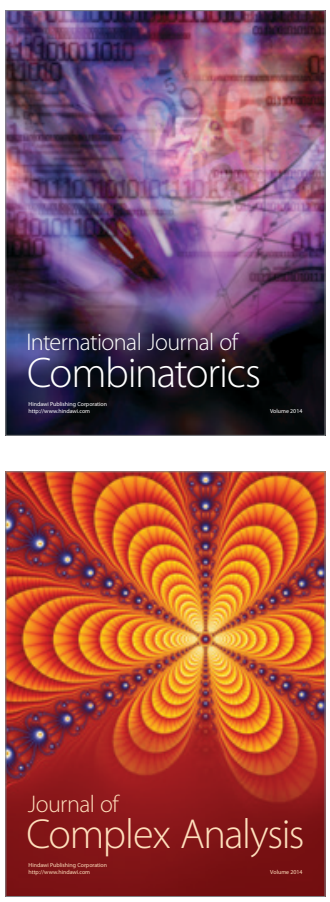

International Journal of

Mathematics and

Mathematical

Sciences
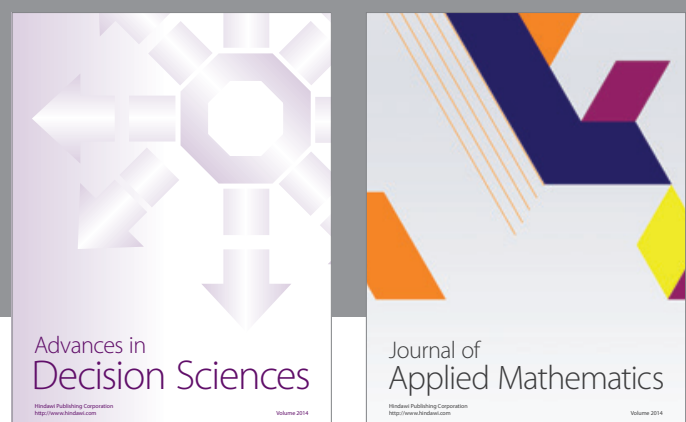

Journal of

Applied Mathematics
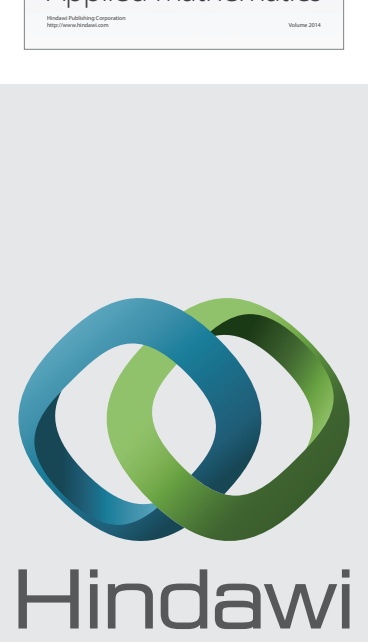

Submit your manuscripts at http://www.hindawi.com
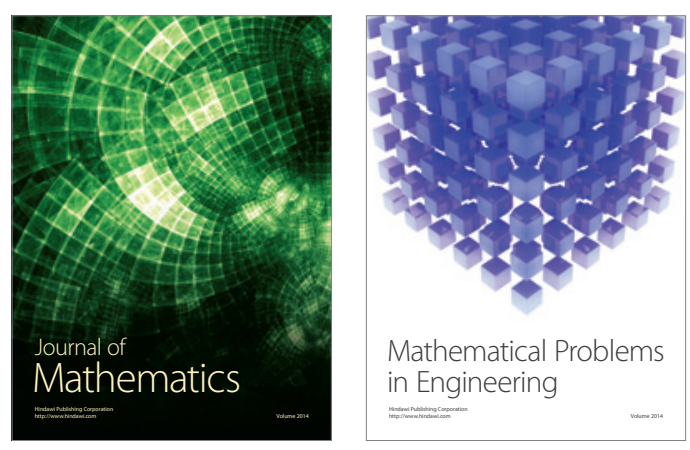

Mathematical Problems in Engineering
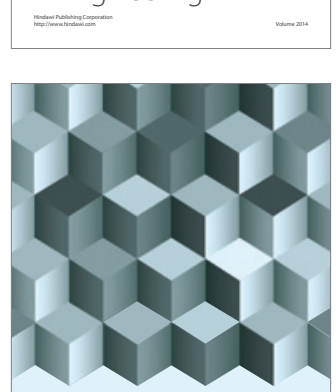

Journal of

Function Spaces
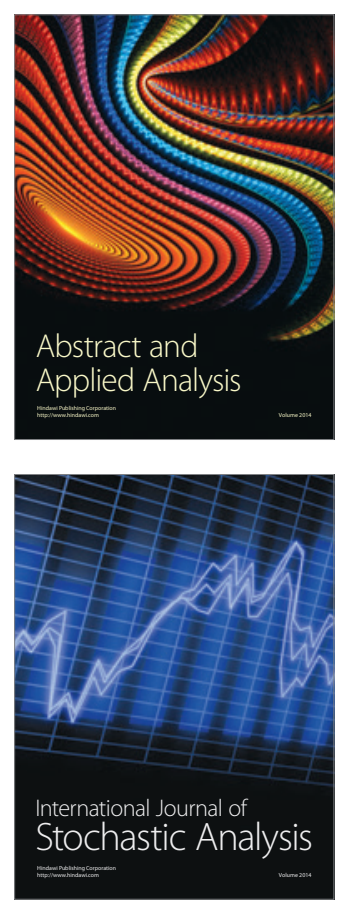

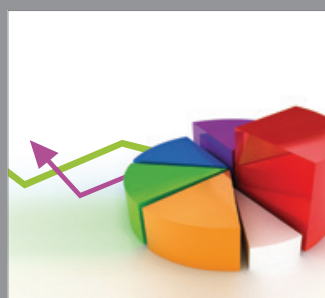

ournal of

Probability and Statistics

Promensencen
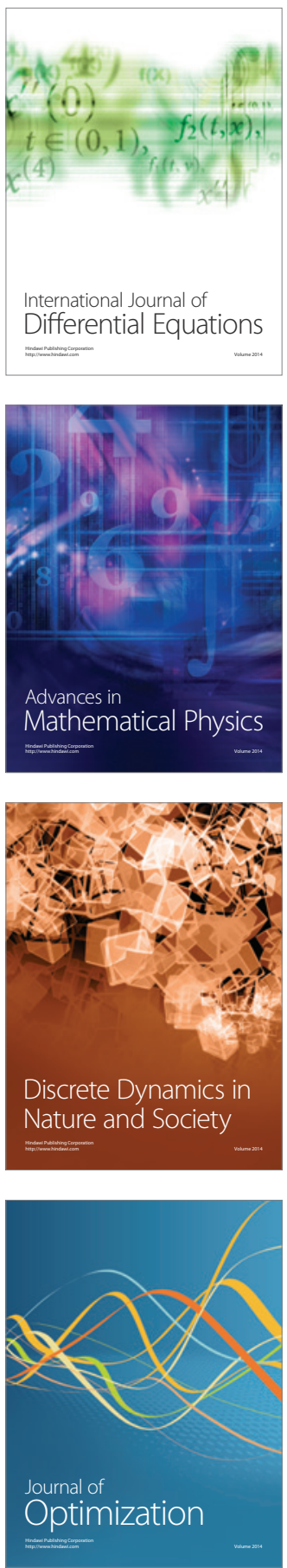